\title{
CXCL12-driven thymocyte migration is increased by thymic epithelial cells treated with prolactin in vitro
}

\author{
Návylla Candeia Medeiros ${ }^{1}$, Felipe Lima Porto $^{1}$, Clarice Agudo de Menezes ${ }^{1}$, \\ Maria Danielma dos Santos Reis ${ }^{1,2}$, Salete Smaniotto ${ }^{1,2}$ and \\ Marvin Paulo Lins ${ }^{1,2} *_{\text {(i) }}$ \\ ${ }^{1}$ Laboratory of Cell Biology, Institute of Biological and Health Sciences, \\ Federal University of Alagoas, Maceió, Brazil \\ ${ }^{2}$ Brazilian National Institute of Science and Technology on Neuroimmunomodulation (INCT-NIM), \\ Oswaldo Cruz Institute, Oswaldo Cruz Foundation, Rio de Janeiro, Brazil \\ *Corresponding author (Email, marvinpaulo@hotmail.com)
}

MS received 18 April 2021; accepted 21 October 2021

\begin{abstract}
The prolactin hormone (PRL), in addition to its known effects on breast development and lactation, exerts effects on the immune system, including pleiotropic effects on the thymus. The aim of this study was to evaluate the influence of PRL on the epithelial compartment of the thymus. Thymic epithelial cells (TECs) (2BH4 cells) and fresh thymocytes were used. Immunofluorescence assay revealed that PRL treatment (10 ng/ $\mathrm{mL}$ ) increases the deposition of laminin and expression of the chemokine CXCL12 in 2BH4 cells. However, no change was observed in the deposition of fibronectin. Moreover, PRL altered F-actin polymerisation, allowing the formation of focal adhesion complexes in treated cells. When 2BH4 cells were pre-treated with PRL, thymocyte adhesion was not altered. However, in the cell migration assay, pre-treatment with PRL potentiated the chemotactic effect of CXCL12 on the migration of total, double-positive, CD4-positive, and CD8-positive thymocytes. Together, the results of this study demonstrate the effect of PRL on thymic epithelial cells, particularly on CXCL12-driven thymocyte migration, confirming that this hormone is a regulator of thymic physiology.
\end{abstract}

Keywords. 2BH4 cell line; PRL; TEC; laminin; fibronectin; F-actin

\section{Introduction}

The thymus is a primary lymphoid organ that provides a specialised microenvironment for the development of $\mathrm{T}$ lymphocytes. In the thymus, bonemarrow-derived precursors undergo a complex differentiation process, which involves sequential expression of various membrane proteins and rearrangement of the genes encoding the T-cell receptor (TCR). The final product interacts with the proteins of the major histocompatibility complex (MHC), together with peptides expressed by the cells of the thymic microenvironment (Miller 1961; Ciofani and Zúñiga-Pflücker, 2007; Zdrojewicz et al. 2016). The thymic microenvironment consists of a threedimensional network of stromal cells comprised of thymic epithelial cells (TECs), macrophages, dendritic cells, endothelial cells, and fibroblasts, as well as soluble components, such as cytokines, chemokines, hormones, and extracellular matrix molecules (ECM). This microenvironment provides the signals required for thymocyte migration, proliferation, survival, and apoptosis during the entire process of $\mathrm{T}$ lymphocyte differentiation (Savino et al. 2004; Petrie and Zúñiga-Pflücker 2007; Nitta and Suzuki 2016).

Among the different types of cells in the thymic microenvironment, TECs are the main component, responsible for the formation of heterogeneous tissues in terms of their morphology, phenotype, and function (Anderson and Takahama 2012). These are found throughout the thymic lobe, both in the cortex (cTECs) 
and in the medulla regions (mTECs), in which they form specific sub-regions. The cTECs and mTECs also express epithelial cell adhesion molecule (EpCAM) in the postnatal thymus, in addition to different cytokeratins. Most mTECs express cytokeratin 5, whereas cTECs express cytokeratin 8 (Alexandropoulos and Danzl 2012). TECs are involved in the development and maturation of thymocytes, secreting factors that are necessary for the dynamic development of the lymphoepithelial compartment. cTECs and mTECs have distinct roles in positive and negative selection processes in the thymus, exhibiting exclusive sets of autoantigens on their surfaces via MHC molecules. Thus, cTECs and mTECs contribute to the generation of diverse and immunocompetent T cells (Alexandropoulos and Danzl 2012).

Several studies have shown that the physiology of the thymus and the differentiation of $\mathrm{T}$ lymphocytes are under a complex neuroendocrine mechanism of control (Lannes-Vieira et al. 1991; Villa-Verde et al. 1995; Smaniotto et al. 2005). This includes both endocrine and paracrine/autocrine pathways, which act on stromal cells and thymocytes through specific receptors. Prolactin (PRL) is a lactogenic hormone synthesised and secreted by cells of the anterior pituitary gland, which function as an important mediator of the immunoneuroendocrine response (Kelley et al. 2007). Moreover, PRL is also produced in extra-pituitary sites, including neurons and cells of the immune system, mainly lymphocytes (Ben-Jonathan et al. 1996; Bole-Feysot et al. 1998; Montgomery 2001). Therefore, it can act both as a hormone, through the classic endocrine pathway, as well as a growth factor, neurotransmitter, or immunoregulator in an autocrine-paracrine manner (Méndez et al. 2005).

In the thymus, PRL and PRL receptors are expressed by stromal cells, such as TECs, and by developing thymocytes (Savino et al. 2016). Once PRL signalling is activated, it induces TEC proliferation, maturation of thymic dendritic cells, and survival and proliferation of early $\mathrm{T}$ cell precursors, and inhibits thymocyte apoptosis after exposure to glucocorticoids (Dardenne et al. 1989; Carreño et al. 2004, 2005; Krishnan et al. 2013). However, our understanding of the action of PRL on thymic physiology remains limited. To contribute towards the study of neuroendocrine control over the thymus, we investigated the influence of prolactin on thymic epithelial cells and its participation in the TEC-thymocyte interactions.

\section{Materials and methods}

\subsection{Culture of thymic epithelial cells (2BH4 TECs)}

The cell line of TECs, namely 2BH4, derived from the thymus of C57BL/6 mice, was used. These cells have a mixed phenotype, expressing cortical and medullary TEC markers (Werneck et al. 2000). 2BH4 cells were cultivated in 25 or $75 \mathrm{~cm}^{2}$ culture flasks with RPMI 1640 medium, supplemented with $10 \%$ foetal bovine serum (FBS), 1\% L-glutamine, and $40 \mathrm{mg} / \mathrm{mL}$ gentamicin (all from Invitrogen, Carlsbad, CA, USA) (designated as complete RPMI medium). The cells were maintained in sterile conditions in an incubator at $37^{\circ} \mathrm{C}$ in a humidified atmosphere containing $5 \% \mathrm{CO}_{2}$. These cells were passaged when they were in a state of semiconfluence $(80 \%)$ by treating with $0.25 \%$ trypsin solution and $0.02 \%$ EDTA in a calcium- and magnesiumfree solution ( $\mathrm{pH}$ 7.2; Gibco, Waltham, MA, USA).

\subsection{Obtaining fresh thymocytes}

Fresh thymocytes were obtained from C57BL/6 mice aged 4-8 weeks (male or female) provided by Central Biotherium of UFAL. These animals were housed under controlled temperature $\left(22 \pm 2^{\circ} \mathrm{C}\right)$ with a $12 \mathrm{~h}$ light/dark cycle and free access to water and food. After euthanasia, the thymuses were removed, mechanically disrupted, and added to a 24-well plate containing $1 \mathrm{~mL}$ of $4 \%$ FBS in phosphate buffer solution (PBS) (Sigma-Aldrich, St. Louis, MO, USA) to release thymocytes (all procedures were approved by the Institutional Ethical Committee on Animal Experimentation under protocol number 14/2015 CEUA/ UFAL). The supernatant was collected, and the cells were counted in a Neubauer chamber using the exclusion method with $0.02 \%$ Trypan blue solution (Sigma-Aldrich).

\subsection{Prolactin treatment}

2BH4 cells were plated for $16 \mathrm{~h}$ for cell adhesion and spreading in complete RPMI medium. Next, the medium was replaced, and the cells were treated with PRL 10 ng/mL (Recombinant Mouse Prolactin Protein; RandD systems, Minneapolis, MN, USA) for $24 \mathrm{~h}$ in RPMI medium supplemented with 2\% FBS (Carreño et al. 2005). 


\subsection{Flow cytometry}

Cell suspensions $\left(1 \times 10^{6}\right)$ of $2 \mathrm{BH} 4$ cells or thymocytes were evaluated for the expression of surface molecules using the following fluorochrome-conjugated antibodies: anti-pan-cytokeratin/Alexa Fluor 488, anti-CD49e/PE, anti-CD49f/FITC (all from eBioscience, San Diego, CA, USA), anti-CD304/APC (Invitrogen), anti-CD184/PE, anti-MHC I/FITC, anti$\mathrm{MHC}$ II/PE, anti-CD4/APC, and anti-CD8/PerCP (all from BD Biosciences, San Diego, CA, USA). The control isotypes IgG1, IgG2a, and IgG2b (conjugated to their respective fluorochromes) were used as negative controls. After 20 min incubation for cell staining (at $4{ }^{\circ} \mathrm{C}$ in the dark), the cells were washed with PBS containing 4\% FBS, centrifuged, and fixed with $2 \%$ formaldehyde (VETEC, Duque de Caxias, RJ, Brazil). Analysis was performed using a flow cytometer (FACSCanto II, BD Biosciences), and the data were analysed using WinMDI software version 2.8.

\subsection{Scanning electron microscopy (SEM)}

In a 24-well plate, circular slides were plated and coated with $0.1 \%$ bovine serum albumin (BSA; SigmaAldrich) for $60 \mathrm{~min}$. After removing this solution, 2BH4 cells $\left(2 \times 10^{4}\right)$ were plated with $500 \mu \mathrm{L}$ of complete RPMI. After $16 \mathrm{~h}$ of incubation, the cultures were washed with PBS and fixed with $0.5 \%$ glutaraldehyde (VETEC) in PBS (Lins et al. 2020). Images were collected using a large-field secondary electron detector in low-vacuum mode. Prior to analysis, the samples were sputtered with gold for $10 \mathrm{~s}$. Image acquisition was performed at an accelerating voltage of $15 \mathrm{kV}$, according to the method described by Souza et al. (2014). SEM was carried out using a Superscan SSX-550 electron microscope (Shimadzu, Japan).

\subsection{Immunofluorescence assay}

$2 \mathrm{BH} 4$ cells $\left(2 \times 10^{4}\right.$ cells $)$ were plated in an 8-well LabTek Chamber Slide ${ }^{\mathrm{TM}}$ System (Nunc, NY, USA) for 16 $\mathrm{h}$, treated with PRL for $24 \mathrm{~h}$, washed in PBS, fixed with methanol (Synth, Diadema, SP, Brazil) for $10 \mathrm{~min}$, and subjected to an indirect immunofluorescence assay. TECs were pre-incubated for 30 min with PBS containing $1 \% \mathrm{BSA}$, followed by the addition of primary antibodies such as anti-fibronectin, anti-laminin (both from Sigma-Aldrich), and anti-CXCL12 (eBioscience) for $1 \mathrm{~h}$ in a humid chamber at room temperature $\left(25^{\circ} \mathrm{C}\right)$. Next, the cells were washed three times with PBS and incubated for 45 min with FITC-conjugated goat antirabbit secondary antibody (Sigma-Aldrich). Subsequently, the cells were washed again in PBS, and the slides were mounted in glycerol/PBS (1:3). As a negative control, the cells were not incubated with the primary antibody, but only with the secondary antibody, so as not to produce any signal. After covering with a glass coverslip, the slides were examined using fluorescence microscopy (Nikon Eclipse 50i; Nikon Instruments Inc., Chicago, IL, USA). The fluorescence intensity was determined in pixels and quantified using Image $\mathbf{J}$ version 1.44 (NIH, MD, USA).

\subsection{Direct F-actin cytoskeleton staining}

2BH4 cells $\left(2 \times 10^{4}\right)$, treated with PRL as described above, were washed in PBS, fixed in paraformaldehyde $(4 \%)$ in PBS, and permeabilised with $0.5 \%$ Triton $\mathrm{X}-100$ for $5 \mathrm{~min}$. The cells were stained for F-actin by direct staining with FITC-conjugated phalloidin (Sigma-Aldrich) for $1 \mathrm{~h}$. Subsequently, the cells were washed in PBS, and mounted in glycerol and PBS (1:3). Modifications in the cytoskeleton were evaluated using fluorescence microscopy, and photomicrographs were obtained using a Nikon camera model DS-Ri1 (Nikon Eclipse 50i; Nikon). The fluorescence intensity and cellular area were determined in pixels and quantified using ImageJ version 1.44.

\subsection{Cell adhesion assay}

The effect of PRL on adhesive capacity of thymocytes in TECs was assessed using a cell adhesion assay. Briefly, $2 \times 10^{4} 2 \mathrm{BH} 4$ cells were grown in $25 \mathrm{~cm}^{2}$ flasks in the presence of complete RPMI medium for $16 \mathrm{~h}$ in a $\mathrm{CO}_{2}$ atmosphere at $37^{\circ} \mathrm{C}$ for cell adhesion and spreading. Subsequently, the cells were treated or not (control) with PRL $10 \mathrm{ng} / \mathrm{mL}$ for $24 \mathrm{~h}$. After treatment, the medium was replaced and 2BH4 cells were cocultured for $1 \mathrm{~h}$ with fresh thymocytes (in a proportion of 50 thymocytes/1 TEC) to allow for heterocellular adhesion interaction. Next, the non-adherent thymocytes were gently removed by washing with PBS at room temperature. The thymocytes that adhered to the TECs were collected by washing with cold PBS and counted. To determine the phenotype of the adhered thymocytes, they were stained with anti-CD4/anti-CD8 antibodies for analysis by flow cytometry. 


\subsection{Thymocyte migration towards 2 BH4 TECs}

Thymocyte migration assays were performed using a transwell system (Corning Costar, Cambridge, MA, USA), with inserts of $6.5 \mathrm{~mm}$ diameter, in polycarbonate membranes, and with $5 \mu \mathrm{m}$ pores. Initially, $2 \mathrm{BH} 4$ cells were plated at $10^{4}$ cells/well, in a 24 -well plate in the presence or absence of PRL $(10 \mathrm{ng} / \mathrm{mL})$ in complete RPMI medium $(500 \mu \mathrm{L})$ and maintained at $37^{\circ} \mathrm{C}$ in a $5 \% \mathrm{CO}_{2}$ atmosphere for $24 \mathrm{~h}$. The inserts were then incubated for $45 \mathrm{~min}$ with PBS- $0.1 \%$ BSA solution, placed into wells of 24-well plates, and freshly obtained thymocytes $\left(2 \times 10^{6}\right)$ were added to the upper chamber. Notably, the TEC medium was not replaced before the co-culture. In some wells, CXCL12 (200 ng/mL, RandD Systems) was added to the lower chamber, on the $2 \mathrm{BH} 4$ cultures. After $3 \mathrm{~h}$, the migrating cells, deposited in the lower chamber, were collected and counted. To determine the thymocyte subsets, these cells were subjected to cytofluorimetric analysis.

\subsection{Statistical analysis}

Data are presented as the mean \pm standard error of the mean (SEM). Student's $t$-test or one-way ANOVA, followed by Tukey's test, were used for statistical analysis. Values of $\mathrm{p} \leq 0.05$ were considered statistically significant. Statistical analysis was performed and graphs were generated using GraphPad Prism 5.0 (GraphPad Software Inc., La Jolla, CA, USA).

\section{Results}

\subsection{Characterisation of TEC line}

The cell line used in this study was isolated by Amarante-Mendes et al. (1995) and characterised as TECs. To confirm this, the characteristics of these cells were evaluated using scanning electron microscopy. As a result, the $2 \mathrm{BH} 4$ cells were confirmed to have a typical epithelial appearance, and a polyhedral and irregular morphology, with cytoplasmic extensions, membrane undulations, and centralised nuclei (figure 1A). In addition to this initial characterisation, we evaluated the expression of some molecules with critical epithelial functions. As demonstrated in figure $1 \mathrm{~B}, 97.99 \%$ of the cells were positive for cytokeratin (CK), highlighting their epithelial phenotype. Moreover, these cells expressed the receptors
VLA-5 (99.95\%), VLA-6 (80\%), neuropilin-1 (NRP-1) (55.37\%), MHC-I (54.29\%), and MHC-II (7.99\%). Thus, our cellular model was well characterised as TEC.

\subsection{CXCL12 production was enhanced after PRL treatment}

Among the thymic soluble proteins, the chemokine CXCL12 is the most extensively studied protein for its effects on thymocyte migration, and TECs are the main producers of this factor (Lucas et al. 2017). Therefore, we investigated whether PRL $(10 \mathrm{ng} / \mathrm{mL})$ could modulate CXCL12 production by $2 \mathrm{BH} 4$ cells. An immunofluorescence assay showed a significant increase (CTR: $5285 \pm 377$ vs. PRL: $11910 \pm 734$, in MFI units, $\mathrm{p}<0.001)$ in chemokine production by 2BH4 TECs when the cells were treated with PRL (figure 2). This increase was observed through qualitative (2A) and quantitative (2B) analyses of the cells.

The thymic interrelationship between chemokines and ECM molecules has already been established regarding the potentiation of thymocyte migration, since both act synergistically (Savino et al. 2015). Thus, after we attested the augmentation of CXCL12 by PRL treatment, we investigated the role of this hormone in the modulation of thymic ECM.

\subsection{Prolactin modulates ECM deposition by $2 \mathrm{BH} 4$ TECS}

ECM directly influences the normal functioning of TECs. These molecules facilitate the diffusion of secretory products and enable cell anchorage of thymocytes for further differentiation (Hun et al. 2017). To evaluate the influence of PRL on ECM deposition in 2BH4 TECs, an immunofluorescence assay was performed with specific markers for fibronectin (FN) and laminin (LM).

Qualitative (figure 3A) and quantitative (figure 3B) analyses showed that PRL treatment did not alter FN deposition. Regarding LM production, qualitative analysis showed an increase of this glycoprotein by 2BH4 TECs (figure 3C), and quantification of fluorescence intensity confirmed that the treatment enhanced (CTR: $18.97 \pm 1.23$ vs. PRL: $23.54 \pm 1.43$, in MFI units, $\mathrm{p}<0.05)$ the LM deposition when compared to the control group (figure 3D).

The ECM is connected via integrins to the actin cytoskeleton, thereby exerting important functions in 
(A)
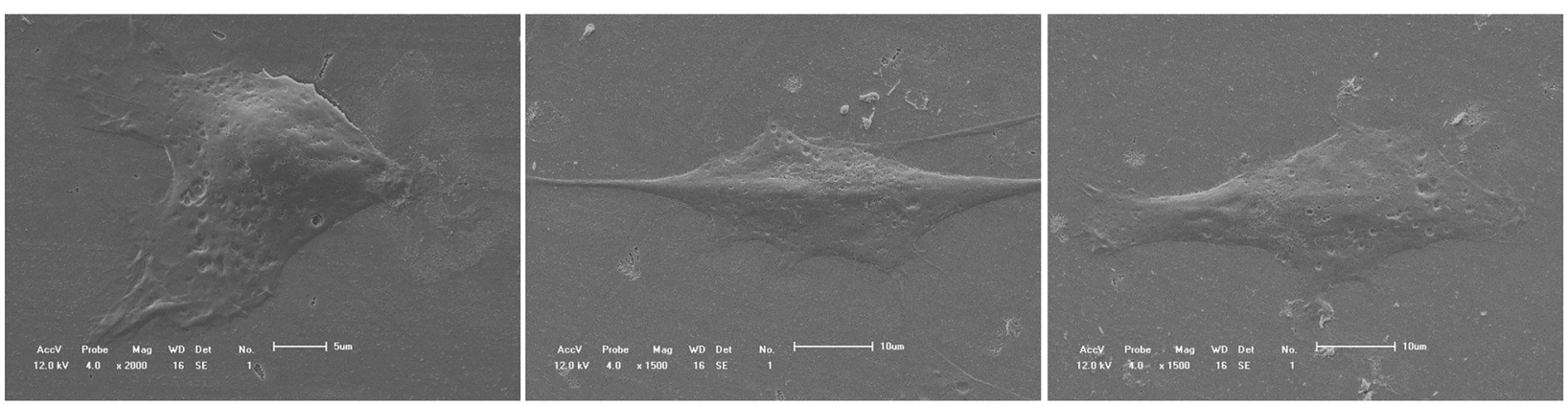

(B)

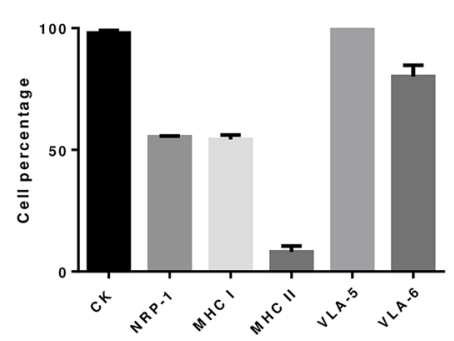

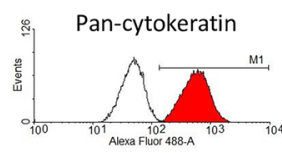
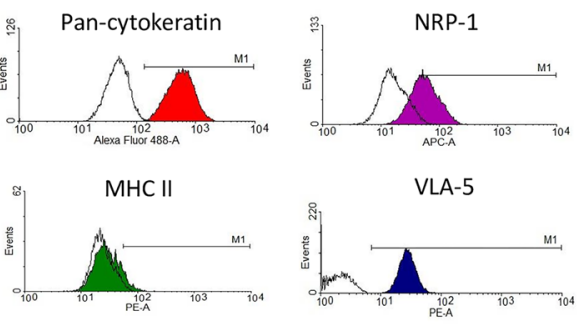
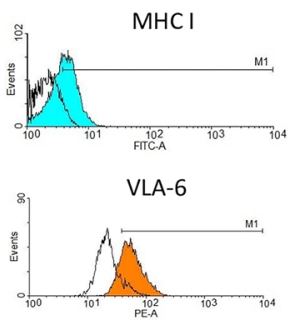

Figure 1. Characterisation of the $2 \mathrm{BH} 4$ cells. (A) SEM images showing morphological phenotype of $2 \mathrm{BH} 4$ TECs plated in 2D arrangement. The cells exhibit typical epithelial appearance, with irregular and polyhedral morphology, discrete membrane extensions, and undulations (magnification: 2000×). (B) Functional characterisation by flow cytometry demonstrating the expression of pan-cytokeratin (CK), neuropilin-1 (NRP-1), MHC I and -II molecules, VLA-5 and -6 integrins in 2BH4 cells. The bars graphic (left) indicates the percentage of positive cells for these cell markers $(n=6$; twoindependent experiments). Representative histograms (right) of the fluorescence intensity show the specific staining (colour curve) and Ig isotype control (white curve).

(A)

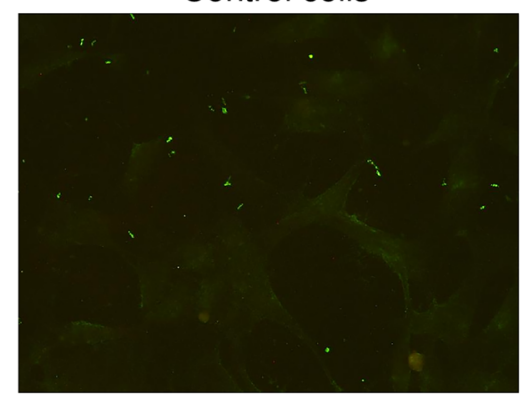

Treated cells

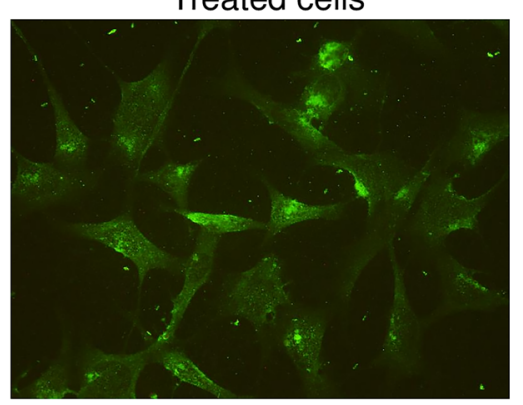

(B)

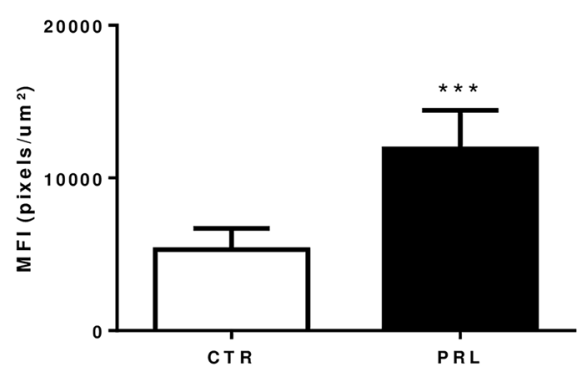

Figure 2. PRL-treated 2BH4 TECs modulate CXCL12 production positively. (A) Photomicrographs demonstrating the expression of CXCL12, stained in green, by 2BH4 cells after $24 \mathrm{~h}$ of treatment with PRL. As observed, PRL improved CXCL12 production by these cells. Images show higher staining in green, indicating more CXCL12 in TECs. Magnification: 400×. (B) Bar graphs showing the quantification of CXCL12 deposition, using ImageJ, in mean fluorescence intensity (MFI). Each column represents the mean \pm SEM obtained from two independent experiments, $n=12$ in each group, ${ }^{* * *} p<0.001$.

cell behaviour and physiology (Gkretsi and Stylianopoulos 2018). Since we observed changes in the ECM evoked by PRL, it was in our interest to evaluate the F-actin content in treated cells.

\section{$3.4 \quad$ F-actin cytoskeleton is a target of the prolactin effects}

The actin cytoskeleton provides mechanical support for organisation of the cellular structure, and its reorganisation generates dynamic changes in cell shape and in cell-cell interactions (Phee et al. 2014). Direct labelling of F-actin by phalloidin was used to analyse the morphology and disposition of the actin filaments in the cytoplasm of 2BH4 cells.

In the control group, the cells presented a rounded morphology, scarce cytoplasmic volume, and evident filopodia. In the PRL-treated group, the cells exhibited a longer elongated F-actin cytoskeleton, spindle-shaped morphology, more condensed cytoplasm, and evident focal adhesion complexes (figure 4). Furthermore, 
Control cells

(A)

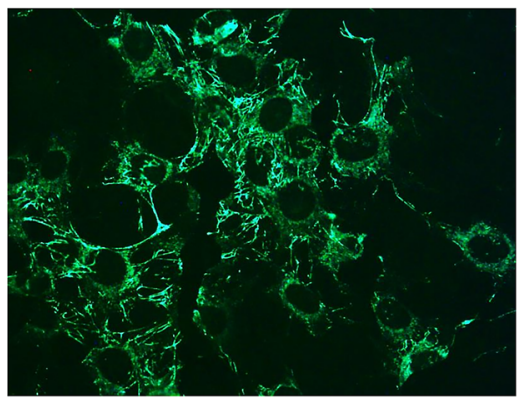

(C)

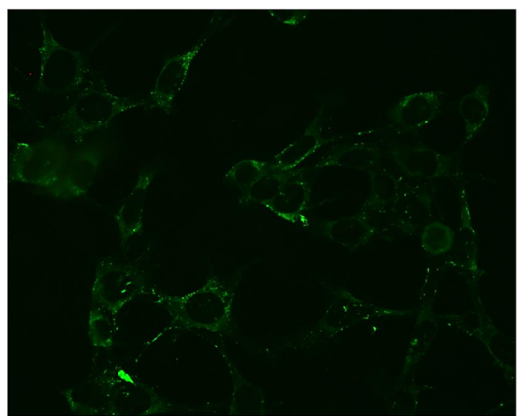

Treated cells
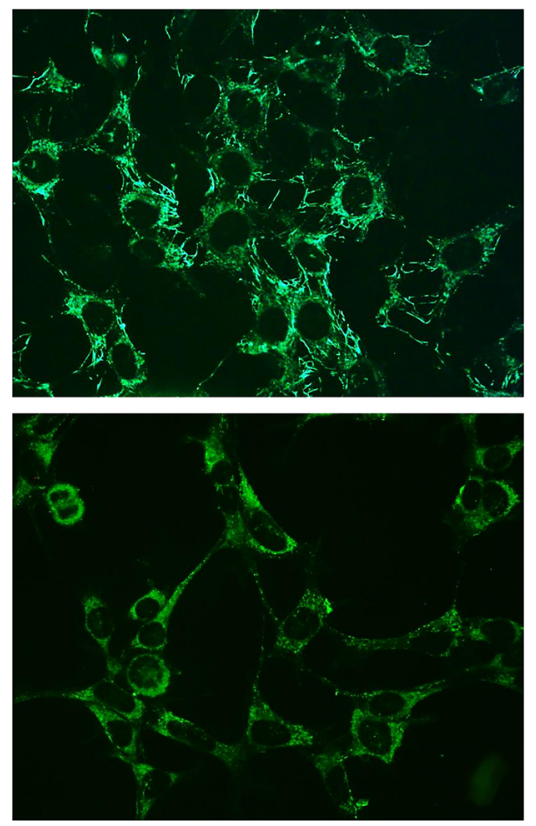

(B)

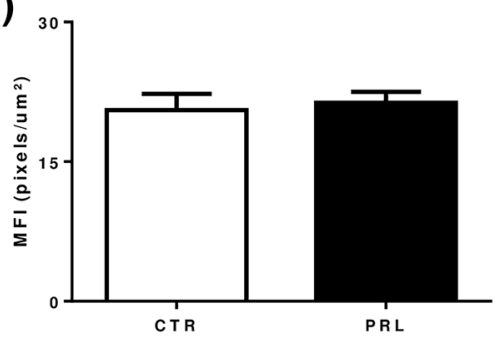

(D)

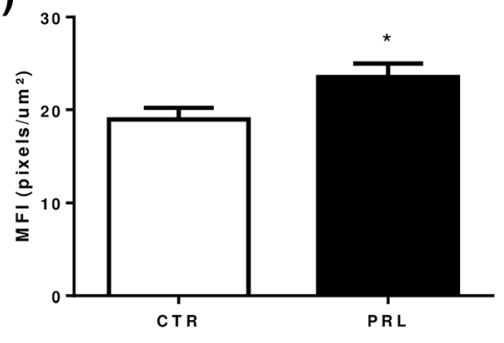

Figure 3. ECM deposition by $2 \mathrm{BH} 4$ cells after PRL treatment. TECs were responsive to PRL and modulated the production of LM, but not of FN. Photomicrographs showing the deposition of FN (A) and LM (C), stained in green. Only LM had a gain in its production by $2 \mathrm{BH} 4$ TECs after treatment with PRL. FN production was unchanged. Magnification: $400 \times$. Bars demonstrating the quantitative analysis of FN (B) and LM (D) deposition, using ImageJ, in mean fluorescence intensity (MFI). Each column represents the mean \pm SEM obtained from two independent experiments, $n=12$ in each group, ${ }^{*} p<0.05$.
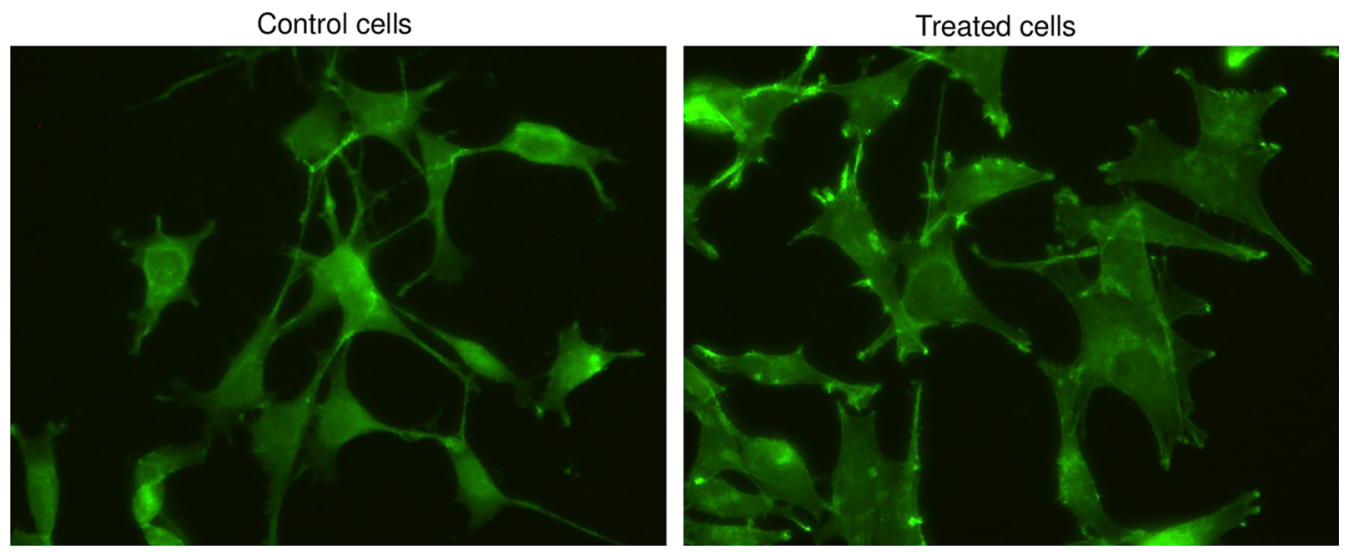

Figure 4. 2BH4 F-actin cytoskeleton is reorganised after PRL treatment. Representative photomicrographs of the control cells and cells treated with PRL. Using phalloidin, it was possible to stain the cytoskeleton (green) and observe the changes in lamellipodia, filopodia and focal adhesions. Furthermore, cellular area was increased by PRL treatment compared with control cells (46\% of augmentation). Magnification: $400 \times$.

cellular area was evaluated by ImageJ quantification, and treated cells were more enlarged $\left(4811 \pm 426 \mu \mathrm{m}^{2}\right.$ **) than control cells $\left(3293 \pm 210 \mu \mathrm{m}^{2}\right)$.

Thus far, the evidence indicated that PRL has significant effects on TEC biology, supporting the argument that this hormone may influence the interaction of TECs with thymocytes. Therefore, functional cell assays were employed in subsequent experiments.

\subsection{TEC-thymocyte adhesion after PRL treatment}

ECM ligands and receptors (as well as F-actin content) have been shown to play a role in TEC-thymocyte interactions, which occur through classical adhesion molecules (Gameiro et al. 2010). Thus, whether PRL is able to modulate the degree of thymocyte adhesion to TECs in in vitro co-cultures was investigated. 
Pre-treatment of 2BH4 TECs with PRL did not affect the number of total thymocytes that adhered to epithelial cells compared with the control group, nor did it change the number of cells in the different subsets of thymocytes (figure 5).

Although the thymocyte adhesion levels were not altered by treatment with prolactin, the possibility that this hormone acts on the migratory capacity of these cells was not ruled out. Therefore, the last set of experiments were used to evaluate this parameter.

\subsection{CXCL12-driven migration of thymocytes is influenced by PRL-treated $2 \mathrm{BH} 4$ cells}

Given the importance of thymocyte migration in thymic function, and driven by the lack of studies on the effects of PRL in this process, we evaluated thymocyte migration towards conditioned medium of 2BH4 cells treated with PRL. As shown in figure 6A, PRL pretreatment did not increase the total number of migrating thymocytes compared to those in the control group. However, as expected, the presence of the CXCL12 chemokine in the conditioned medium increased the total number of migrating cells. Interestingly, a significant augmentation in the migration of total thymocytes was observed in the conditioned medium of PRL- treated 2BH4 TECs in combination with CXCL12. Importantly, the treatment of thymocytes with PRL for up to 3 hours did not alter the expression levels of the CXCL12 receptor, CXCR4, in vitro.

Regarding the migrating subsets of thymocytes (figure 6B), an increase in the number of migrating cells (double positive and $\mathrm{CD} 8^{+}$thymocytes) in the groups exposed to CXCL12 alone or in combination with the PRL-conditioned medium was observed compared to the control and PRL group. Moreover, in the $\mathrm{CD}^{+}$subset, a significant increase in the cell migration of cells in the PRL + CXCL12 group was observed than in the control group.

\section{Discussion}

In this study, the participation of PRL in the stromal compartment of the thymus was evaluated. Specifically, we aimed to understand the role of this hormone in the physiology of TECs. Our results showed that PRL affects 2BH4 TECs, as demonstrated by the modulation of the expression of extracellular matrix ligands and CXCL12, reorganisation of the cytoskeleton, and the increase in the number of migrating thymocytes.
Total thymocytes

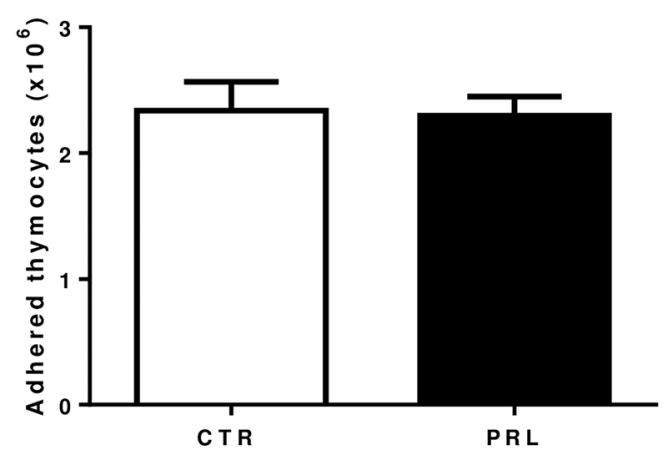

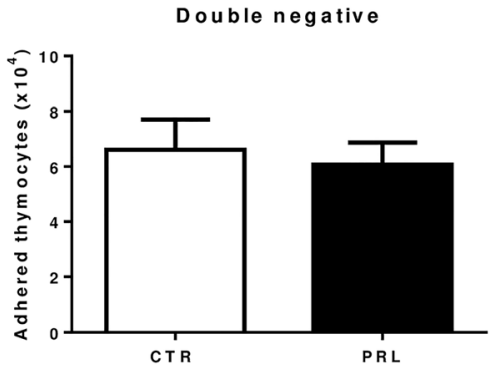

CD $4^{+}$Thymocytes

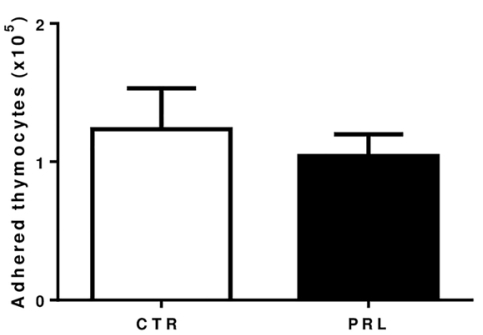

Double positive

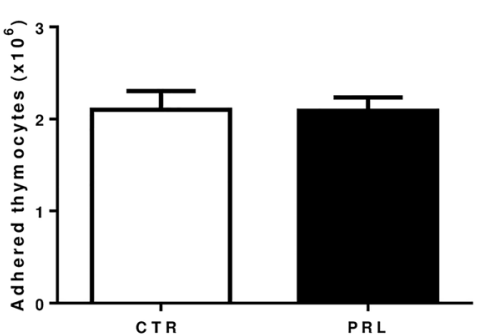

CD $8^{+}$Thymocytes

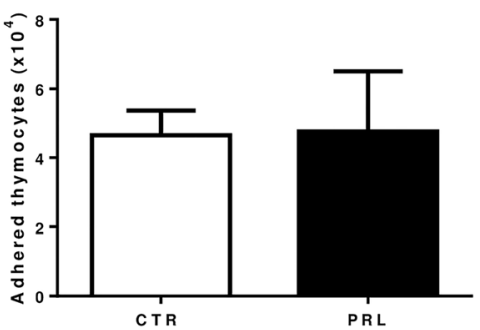

Figure 5. PRL maintains thymocyte adhesion to $2 \mathrm{BH} 4$ cells. TECs were pre-treated with PRL (10 ng/mL) for $24 \mathrm{~h}$. After replacement of medium, thymocytes were submitted to adhesion for $1 \mathrm{~h}$ on $2 \mathrm{BH} 4 \mathrm{TEC}$ monolayer. Adherent thymocytes were collected, counted, and marked for cytometry. Absolute number of the counting demonstrated no interference of PRL on thymocyte adhesion to TECs. Bars represent the mean \pm SEM, $n=5$. Statistical analysis was performed using Student's $t$-test. 


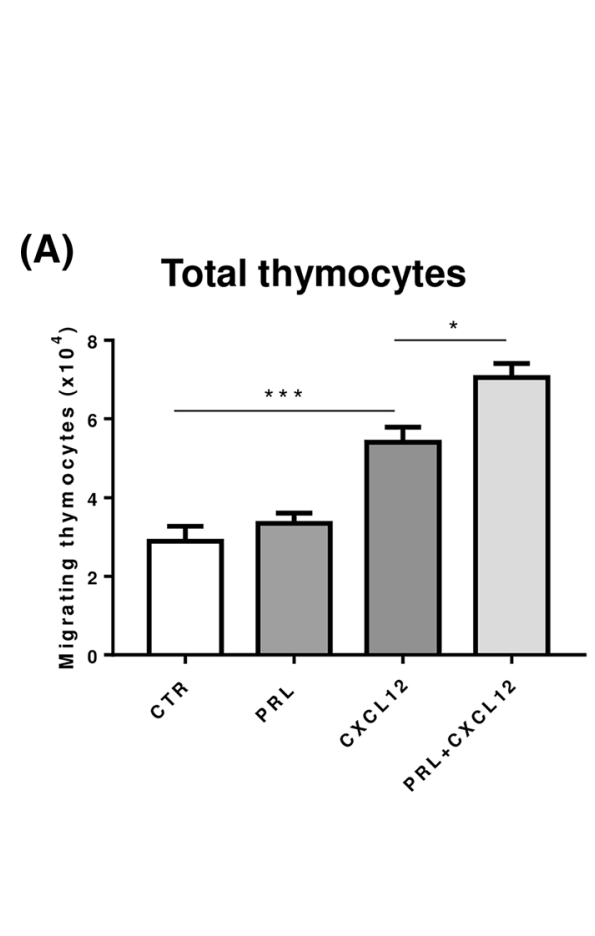

(B)

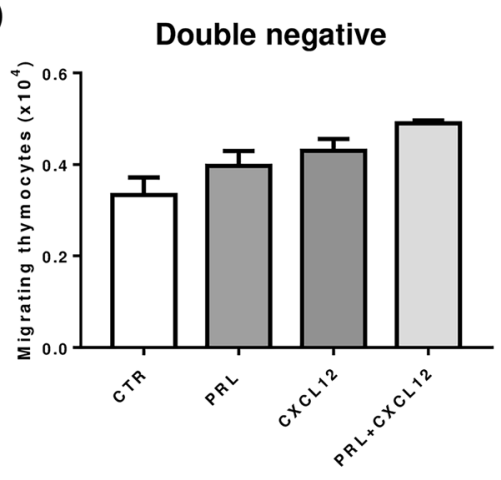

CD4+ Thymocytes

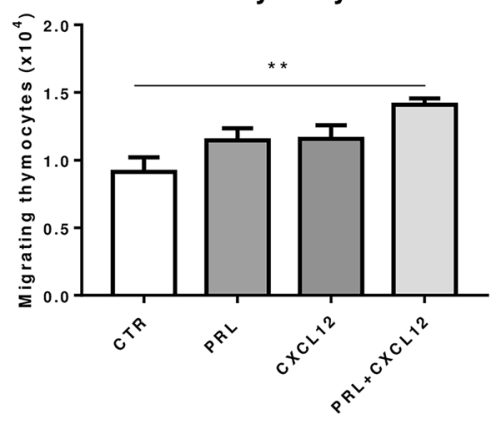

Double positive

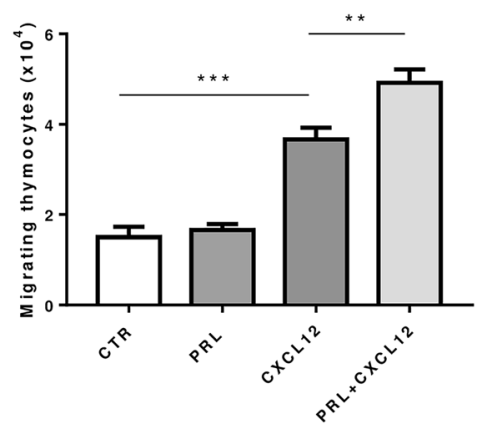

CD8+ Thymocytes

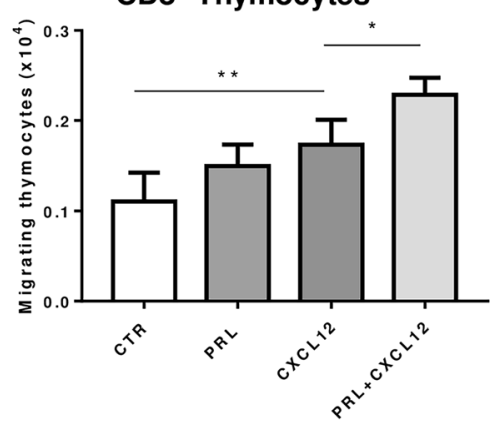

Figure 6. PRL effect on thymocyte migration towards TECs in vitro. $2 \mathrm{BH} 4$ cells were pre-treated with PRL (10 ng/mL) and thymocyte migration (in the same wells with TECs) was carried out for $3 \mathrm{~h}$. Then, migrating thymocytes were recovered by washing and counted to evaluate PRL influence in this phenomenon. (A) Total number of migrating thymocytes. (B) Absolute number of thymocyte subsets defined by CD4/CD8 analysed by flow cytometry. Bars represent mean \pm SEM. Statistical analysis was performed using the ANOVA test followed by Tukey's test, $n=6,{ }^{*} \mathrm{p}<0.05,{ }^{*} p<0.01$ and $* * * p<0.001$.

TECs are the main component of the thymic microenvironment, where they produce soluble factors and express ligands and receptors that direct migration and subsequent lymphocyte maturation (Wang et al. 2020). In this study, we used a TEC cell line obtained from C57BL/6 mice, namely 2BH4 cells. First, this cell line was characterised through morphological analysis by SEM; subsequently the expression of cytokeratins and surface receptors was evaluated by flow cytometry.

2BH4 cells were found to have morphological characteristics compatible with epithelial cells, in agreement with the analysis carried out by AmaranteMendes et al. (1995), who described 2BH4 to have several shapes - from polygonal to fusiform, with small round nuclei with prominent nucleoli. In addition, TECs can be organised as 'cobblestone' or diffuse cells connected by long cytoplasmic extensions. To confirm the epithelial phenotype of these cells, we evaluated the expression of cytokeratin, which is a constituent molecule of the cytoskeleton of epithelial cells, belonging to the intermediate filaments (Almeida $\mathrm{Jr}$ 2004). In our study, a majority of these cells in culture $(97.9 \%)$ exhibited this marker.
Another characteristic found in $2 \mathrm{BH} 4$ cells was the expression of MHC I, MHC II, VLA-5, VLA-6, and NRP-1 surface receptors. 2BH4 expressed both MHC molecules; however, MHC II was expressed at low levels. Studies have shown that MHC I is expressed in large quantities in murine and human TECs, while MHC II displays different levels of expression (Yang et al. 2006; Saldaña et al. 2016; Benhammadi et al. 2020). NRP-1 is a transmembrane glycoprotein that responds to the semaphorin-3A and VEGF family members. This receptor plays a role in embryonic development, angiogenesis, and cell migration. In addition, NRP-1 expression has been detected in thymic stromal cells and TECs, and has been demonstrated to have an effect on thymic physiology (Lins et al. 2020).

The VLA-5 and VLA-6 integrins are heterodimers composed of two chains, $\alpha$ and $\beta$, which mediate interactions between the extracellular environment and actin cytoskeleton. VLA-5 integrin is a receptor for fibronectin and is composed of an $\alpha 5$ chain associated with a $\beta 1$ subunit (Linhares-Lacerda et al. 2010). The VLA- 6 integrin is formed by an $\alpha 6$ chain, which also 
binds to a $\beta 1$ subunit to form the laminin receptor (Golbert et al. 2013). Our results showed that more than $80 \%$ of $2 \mathrm{BH} 4$ cells in culture express these integrins, which are important for migration, adhesion, and differentiation of thymocytes on the substrate of extracellular matrix.

A clear comparison between 2BH4 TECs and primary TECs should be described here. Primary TEC cultures can be readily obtained from mouse or human thymus, applying enzymatic dissociation associated with FACS- or bead-sorted methods to purify TECs. These cells kept the phenotypic, physiologic and functional features, however they had decreased cell viability due prolonged time of culture (Villegas et al. 2018). In our study, we extended the detailed characterisation written by Amarante-Mendes et al. (1995) and reinforced the cellular model for TEC of 2BH4 cells.

The chemokine CXCL12 has pleiotropic effects, acting in the migration of $\mathrm{T}$ cell precursors for specific microenvironments, wherein there are basic factors present for their development. This chemokine is selectively expressed in most thymic stromal cells (Lucas et al. 2017). Our results demonstrated the modulation of CXCL12 production by PRL treatment in 2BH4 TECs in vitro. Consistent with these results, other hormones are also able to modulate the expression of chemokines. For example, treatment with growth hormone $(\mathrm{GH})$ increases the expression of CXCL12 in thymic nurse cells and in the thymic microenvironment (Smaniotto et al. 2005).

PRL treatment promoted an increase in the production of the LM glycoprotein. The deposition of LM in the thymus is under hormonal regulation, such as $\mathrm{GH}$, which increases the deposition of LM in TECs in vitro, or under the regulation of triiodothyronine (T3), which enhances intrathymic expression of LM. Also, Sema$3 \mathrm{~A}$ increases LM production in thymic stromal cells (Lins et al. 2020). Similarly, mice that were administered intraperitoneal glucocorticoids (GCs) exhibited a greater amount of LM within the thymic lobes. Interestingly, some pathological conditions such as type 1 diabetes and infections by Trypanosoma cruzi and Plasmodium berguei also exacerbate the distribution and density of LM in the thymus (Savino et al. 2015).

Next, the actin cytoskeleton, the main support of cells and the organisation of which is essential for cell movement, was evaluated. Its interaction with integrins and focal adhesion complexes facilitates cell migration. After PRL treatment, 2BH4 cells were found to form structures including focal adhesion complexes, lamellipodia, and filopodia, which are key to the migration process. These changes in the cytoskeleton, caused by PRL, agree with those in a study by Da Silva et al. (2015), in which PRL changed the distribution of F-actin filaments in mammary cells. This type of remodelling promoted a greater migration of cells.

Lymphocyte migration depends on sequential cell adhesion/de-adhesion events, in which ECM proteins are involved. We showed that PRL did not interfere with thymocyte adhesion to 2BH4 cells. Previous studies have shown that the treatment of TECs (IT$76 \mathrm{M} 1$ cell line) from BALB/c mice with PRL $\left(10^{-8} \mathrm{M}\right.$, for $24 \mathrm{~h}$ ) promoted an improvement in thymocyte adhesion, due to the increased amounts of ECM components and their respective integrins (De MelloCoelho et al. 1997). However, further studies are required to better understand the role of PRL in TECthymocyte adhesion. This invariant adhesion may be due to the short contact time $(1 \mathrm{~h})$ between cells, or the greater amount of LM was not high enough to retain more thymocytes on the surface of the 2BH4 cells.

The final experiment analysed thymocyte migration towards 2BH4 TECs pre-treated with PRL. Our results showed that PRL acted on TECs to increase the total number of CXCL12-driven migrating thymocytes. Smaniotto et al. (2005) showed that thymocytes derived from GH-transgenic mice migrated more efficiently through a transwell chamber in the presence of CXCL12. In addition, the transendothelial migration of thymocytes pre-treated with $\mathrm{GH}$ was increased in response to CXCL12 (Smaniotto et al. 2011). These results contribute to the advancement of knowledge related to thymic physiology, demonstrating, for the first time, the effect of PRL on CXCL12-driven migration, thus opening a new field of scientific investigation.

Taken together, the data presented in this study provide an insight into the effects of PRL on the thymic microenvironment, particularly on TEC interaction with soluble factors, as the chemokine CXCL12 was found to be important for the migration of developing thymocytes during differentiation.

\section{Acknowledgements}

We wish to honor Navylla Candeia Medeiros for her tireless work on PRL and thymus during her PhD. Unfortunately, her life was abruptly interrupted by COVID-19, leaving us orphans of her joy and love. We miss her. May her memory be perpetuated in each one of us. We thank the laboratory technicians Ana Rubia Batista Ribeiro (Physics Institute of Federal University 
103 Page 10 of 11

of Alagoas) and Juliane Pereira da Silva for assistance in image acquisition on the Scanning Electron Microscope and for handling of flow cytometer, respectively. Also, we are grateful to Erick Gabriel Alves Ferreira for improving the resolution of the figures.

\section{Funding}

The study was supported by Brazilian National Council for Scientific and Technological Development - CNPq (No. 408677/2016-3 and No. 304408/2018-2) and Foundation for Funding Research in the State of Alagoas - FAPEAL (No. 60030 001260/2017).

\section{References}

Alexandropoulos K and Danzl NM 2012 Thymic epithelial cells: antigen presenting cells that regulate $\mathrm{T}$ cell repertoire and tolerance development. Immunol. Res. 54 177-190

Almeida Junior HL 2004 Cytokeratins. An. Bras. Dermatol. 79 135-145

Amarante-Mendes JG, Chammas R, Abrahamsohn P, Patel PC, Potworowski EF and Macedo MS 1995 Cloning of a thymic stromal cell capable of protecting thymocytes from apoptosis. Cell Immunol. 161 173-180

Anderson G and Takahama Y 2012 Thymic epithelial cells: working class heroes for $\mathrm{T}$ cell development and repertoire selection. Trends Immunol. 33 256-263

Benhammadi M, Mathé J, Dumont-Lagacé M, Kobayashi KS, Gaboury L, Brochu S and Perreault C 2020 IFN- $\lambda$ enhances constitutive expression of MHC Class I molecules on thymic epithelial cells. J. Immunol. 205 1268-1280

Ben-Jonathan N, Mershon JL, Allen DL and Steinmetz RW 1996 Extrapituitary prolactin: distribution, regulation, functions, and clinical aspects. Endocr. Rev. 17 639-669

Bole-Feysot C, Goffin V, Edery M, Binart N and Kelly PA 1998 Prolactin (PRL) and its receptor: actions, signal transduction pathways and phenotypes observed in PRL receptor knockout mice. Endocr. Rev. 19 225-268

Carreño PC, Jiménez E, Sacedón R, Vicente A and Zapata AG 2004 Prolactin stimulates maturation and function of rat thymic dendritic cells. J. Neuroimmunol. 153 83-90

Carreño PC, Sacedón R, Jiménez E, Vicente A and Zapata AG 2005 Prolactin affects both survival and differentiation of T-cell progenitors. J. Neuroimmunol. 160 135-145

Ciofani M and Zúñiga-Pflücker JC 2007 The thymus as an inductive site for T lymphopoiesis. Annu. Rev. Cell Dev. Biol. 23 463-493

da Silva PL, do Amaral VC, Gabrielli V, Montt Guevara MM, Mannella P, Baracat EC, Soares JM Jr and
Simoncini T 2015 Prolactin promotes breast cancer cell migration through actin cytoskeleton remodeling. Front. Endocrinol. 6186

Dardenne M, Savino W, Gagnerault MC, Itoh T and Bach JF 1989 Neuroendocrine control of thymic hormonal production. I. Prolactin stimulates in vivo and in vitro the production of thymulin by human and murine thymic epithelial cells. Endocrinology 125 3-12

de Mello-Coelho V, Villa-Verde DM, Dardenne $M$ and Savino W 1997 Pituitary hormones modulate cell-cell interactions between thymocytes and thymic epithelial cells. J. Neuroimmunol. 76 39-49

Gameiro J, Nagib P and Verinaud L 2010 The thymus microenvironment in regulating thymocyte differentiation. Cell Adh. Migr. 4 382-390

Gkretsi V and Stylianopoulos T 2018 Cell adhesion and matrix stiffness: coordinating cancer cell invasion and metastasis. Front. Oncol. 8145

Golbert DC, Correa-de-Santana E, Ribeiro-Alves M, de Vasconcelos AT and Savino W 2013 ITGA6 gene silencing by RNA interference modulates the expression of a large number of cell migration-related genes in human thymic epithelial cells. BMC Genomics $14 \mathrm{~S} 3$

Hun M, Barsanti M, Wong K, Ramshaw J, Werkmeister J and Chidgey AP 2017 Native thymic extracellular matrix improves in vivo thymic organoid $\mathrm{T}$ cell output, and drives in vitro thymic epithelial cell differentiation. Biomaterials 118 1-15

Kelley KW, Weigent DA and Kooijman R 2007 Protein hormones and immunity. Brain Behav. Immun. 21 384-392

Krishnan N, Thellin O, Buckley DJ, Horseman ND and Buckley AR 2013 Prolactin suppresses glucocorticoidinduced thymocyte apoptosis in vivo. Endocrinology 144 2102-2110

Lannes-Vieira J, Dardenne M and Savino W 1991 Extracellular matrix components of the mouse thymus microenvironment: ontogenetic studies and modulation by glucocorticoid hormones. J. Histochem. Cytochem. 39 1539-1546

Linhares-Lacerda L, Ribeiro-Alves M, Nogueira AC, Mendes-da-Cruz DA, Magalhães DA, Dardenne M, Passos GA and Savino W 2010 RNA interference-mediated knockdown of CD49e ( $\alpha 5$ integrin chain) in human thymic epithelial cells modulates the expression of multiple genes and decreases thymocyte adhesion. BMC Genomics 11 S2

Lins MP, Medeiros NC, Carmo J, Porto FL, Dos Santos Reis MD and Smaniotto S 2020 The Responsiveness of Thymic Stromal Cells to semaphorin-3A. Immunol. Invest. 19 1-16

Lucas B, White AJ, Parnell SM, Henley PM, Jenkinson WE and Anderson G 2017 Progressive changes in CXCR4 expression that define thymocyte positive selection are 
dispensable for both innate and conventional $\alpha \beta \mathrm{T}$-cell development. Sci. Rep. 75068

Méndez I, Cariño C and Díaz L 2005 Prolactin in the immunological system: synthesis and biological effects. Rev. Invest. Clin. 57 447-456

Miller JF 1961 Immunological function of the thymus. Lancet 2 748-749

Montgomery DW 2001 Prolactin production by immune cells. Lupus 10 665-675

Nitta T and Suzuki H 2016 Thymic stromal cell subsets for T cell development. Cell Mol. Life Sci. 73 1021-1037

Petrie HT and Zúñiga-Pflücker JC 2007 Zoned out: functional mapping of stromal signaling microenvironments in the thymus. Annu. Rev. Immunol. 25 649-679

Phee H, Au-Yeung BB, Pryshchep O, O'Hagan KL, Fairbairn SG, Radu M, Kosoff R, Mollenauer M, Cheng $\mathrm{D}$, Chernoff J and Weiss A 2014 Pak2 is required for actin cytoskeleton remodelling, TCR signalling, and normal thymocyte development and maturation. Elife 3 e02270

Saldaña JI, Solanki A, Lau CI, Sahni H, Ross S, Furmanski AL, Ono M, Holländer G and Crompton T 2016 Sonic Hedgehog regulates thymic epithelial cell differentiation. J. Autoimmun. 68 86-97

Savino W, Mendes-Da-Cruz DA, Smaniotto S, Silva-Monteiro E and Villa-Verde DM 2004 Molecular mechanisms governing thymocyte migration: combined role of chemokines and extracellular matrix. J. Leukoc. Biol. 75 951-961

Savino W, Mendes-da-Cruz DA, Golbert DC, Riederer I and Cotta-de-Almeida V 2015 Laminin-mediated interactions in thymocyte migration and development. Front. Immunol. 6579

Savino W, Mendes-da-Cruz DA, Lepletier A and Dardenne M 2016 Hormonal control of T-cell development in health and disease. Nat. Rev. Endocrinol. 12 77-89

Smaniotto S, de Mello-Coelho V, Villa-Verde DM, Pléau JM, Postel-Vinay MC, Dardenne M and Savino W 2005 Growth hormone modulates thymocyte development in vivo through a combined action of laminin and CXC chemokine ligand 12. Endocrinology 146 3005-3017

Smaniotto S, Martins-Neto AA, Dardenne M and Savino W 2011 Growth hormone is a modulator of lymphocyte migration. Neuroimmunomodulation 18 309-313

Souza ST, Agra LC, Santos CE, Barreto E, Hickmann JM and Fonseca EJ 2014 Macrophage adhesion on fibronectin evokes an increase in the elastic property of the cell membrane and cytoskeleton: an atomic force microscopy study. Eur. Biophys. J. 43 573-579

Villa-Verde DM, Mello-Coelho V, Lagrota-Cândido JM, Chammas R and Savino W 1995 The thymic nurse cell complex: an in vitro model for extracellular matrixmediated intrathymic T cell migration. Braz. J. Med. Biol. Res. 28 907-912

Villegas JA, Gradolatto A, Truffault F, Roussin R, BerrihAknin S, Le Panse R and Dragin N 2018 Cultured human thymic-derived cells display medullary thymic epithelial cell phenotype and functionality. Front. Immunol. 23 1663

Wang HX, Pan W, Zheng L, Zhong XP, Tan L, Liang Z, He J, Feng P, Zhao Y and Qiu YR 2020 Thymic epithelial cells contribute to thymopoiesis and $\mathrm{T}$ cell development. Front. Immunol. 103099 (Erratum in: Front. Immunol. $202011628464)$

Werneck CC, Cruz MS, Silva LC, Villa-Verde DM, Savino W and Mourão PA 2000 Is there a glycosaminoglycanrelated heterogeneity of the thymic epithelium? J. Cell Physiol. 185 68-79

Yang SJ, Ahn S, Park CS, Holmes KL, Westrup J, Chang $\mathrm{CH}$ and Kim MG 2006 The quantitative assessment of MHC II on thymic epithelium: implications in cortical thymocyte development. Int. Immunol. 18 729-739

Zdrojewicz Z, Pachura E and Pachura P 2016 The thymus: a forgotten, but very important organ. Adv. Clin. Exp. Med. 25 369-375

Corresponding editor: DIPANKAR NANDI 